Conclusions In assessment for causation of breathlessness, observations of the inspiratory arm of the FVL during MCT may provide clues to the experienced clinician. MCT has potential to be a useful adjunct in the assessment for ILO. However, due to poor inter-rater reliability, further studies are needed to improve our understanding.

Further prospective studies are needed, ideally with MCT and concurrent laryngoscopy to further investigate the utility in assessment for ILO.

\section{S46 LUNG FUNCTION AND PULMONARY SYMPTOMS IN CLASSICAL AND LATE-ONSET FABRY DISEASE}

${ }^{1} \mathrm{AJ}$ Shah, ${ }^{2} \mathrm{~N}$ Shafi, ${ }^{1} \mathrm{~A}$ Saigal, ${ }^{1,2} \mathrm{~S}$ Mandal, ${ }^{1,2} \mathrm{M}$ Lipman, ${ }^{3,4} \mathrm{DA}$ Hughes. ${ }^{1} T$ Thoracic Department, Royal Free London Foundation NHS Trust, London, UK; ${ }^{2}$ UCL Respiratory, London, UK; ${ }^{3}$ Haematology Department, Royal Free London NHS Foundation Trust, London, UK; ${ }^{4} U C L$, London, UK

10.1136/thorax-2021-BTSabstracts.52

Background Fabry Disease (FD) is an X-linked condition resulting from an absolute or relative deficiency of the enzyme alpha-galactosidase A (GLA) leading to a multi-system disorder. FD can be divided into: 1) severe, classical phenotype, most often seen in men; 2) milder late-onset phenotype. While the natural course of classical and late-onset FD has been investigated with regards to cardiac, renal and cerebral manifestations, data concerning pulmonary involvement are limited. This study investigated lung function and symptomatic differences between classical and late-onset FD.

Methods Unselected patients attending Royal Free Hospital, a UK specialist unit for $\mathrm{FD}$, were recruited to a prospective observational study. Baseline demographics and symptom burden were recorded, and all patients underwent lung function testing. Patients were divided into classical and late-onset FD based on the Mount Sinai international FD database. Where this was not possible the lead clinician assessed the phenotype. Results Forty-five FD patients (20 males, 25 females) were recruited with no baseline differences in age, ethnicity, BMI and smoking history. 73\% (33/45) were classical (39\% male, $61 \%$ female) with $27 \%$ late-onset (58\% male, $42 \%$ female) phenotype. $44 \%$ of FD patients had evidence of airway

Abstract S46 Table 1 Phenotypic characterisation with regards to lung function and symptoms

\begin{tabular}{llll}
\hline & Classic Men & $\begin{array}{l}\text { Late-onset men and all females } \\
\text { (Combined Data) }\end{array}$ & P Value \\
\hline N & 13 & 32 & - \\
Age & $39 \pm 11$ & $49 \pm 15$ & 0.03 \\
Lung function (\% predicted) & & \\
FEV1 & $85 \pm 10$ & $92 \pm 12$ & 0.08 \\
FVC & $100 \pm 9$ & $106 \pm 13$ & 0.11 \\
FEV1/FVC & $70 \pm 7$ & $73 \pm 8$ & 0.26 \\
MEF50 & $53 \pm 14$ & $60 \pm 19$ & 0.25 \\
TLCO & $90 \pm 15$ & $88 \pm 21$ & 0.66 \\
KCO & $102 \pm 17$ & $94 \pm 12$ & 0.08 \\
Symptoms & & & 0.03 \\
SOBOE (\%) & $23 \%$ & $59 \%$ & 0.73 \\
Cough (\%) & $46 \%$ & $41 \%$ & 0.82 \\
\hline Wheeze (\%) & $31 \%$ & $34 \%$ & \\
\hline All data parametric, mean \pm SD presented & \\
SOBOE = Shortness of breath on exertion &
\end{tabular}

obstruction based on FEV1/FVC <0.7. As classical FD women resemble late-onset males in their disease pattern we compared classical men and the combined group of late-onset men and all women (table 1). Although the latter group were significantly older, there were no significant differences in ageadjusted lung function. The combined group reported increased breathlessness on exertion (SOBOE) $(59 \%$ vs. $23 \%, \mathrm{p}=0.03)$. However, in subjects with FEV1/FVC $<0.7$ no significant difference in SOBOE were identified $(64 \%$ vs. $22 \%, \mathrm{p}=0.09)$.

Conclusion This study confirms a high prevalence of airway obstruction in FD, with no difference between classical men and our combined group. This appears to be generally mild disease, and does not fully explain the greater frequency of patient-reported breathlessness identified in the combined group. Whilst older age appears relevant, it may also reflect other factors such as concomitant cardiac disease.

\section{REFERENCE}

1. Franzen DP, et al. PloS one. 2017;12(suppl 7):e0180437.

\section{S47 HARD TO SWALLOW; INCIDENCE OF OROPHARYNGEAL DYSPHAGIA IN INDUCIBLE LARYNGEAL OBSTRUCTION (ILO)}

H Lever, K Prior, A Vyas, C Slinger. Lancashire Teaching Hospitals NHS Foundation Trust, Preston, UK

\subsection{6/thorax-2021-BTSabstracts.53}

Introduction Patients diagnosed with ILO may report clinical respiratory symptoms including dyspnoea, inspiratory wheeze and cough (RCSLT 2021). Symptoms indicative of dysphagia (swallowing difficulties) and dysphonia (voice difficulties) have also been reported within this population (Hull et al 2016).

In clinical practice, altered laryngeal sensitivity has been seen in association with patient-reported dysphagia symptoms, in the absence of a mechanical swallowing difficulty.

Aims and Objectives To explore the incidence of reported dysphagia symptoms in patients with a confirmed diagnosis of ILO, and to investigate how many patients were diagnosed with a mechanical oropharyngeal dysphagia. Associated patient co-morbidities were also reviewed.

Methods A retrospective review across a 2 year period (April 2019-2021) was conducted for patients who had been referred through to a Tertiary Airways service, seen for assessment by an SLT and had a confirmed ILO diagnosis via provocation laryngoscopy $(\mathrm{N}=160)$.

Results Of the 160 patients, 52\% ( $\mathrm{N}=82)$ reported symptoms in keeping with oropharyngeal dysphagia. $27 \%(\mathrm{~N}=22)$ of these patients had a clinical bedside swallow assessment by a dysphagia trained SLT. Of these patients, 50\% $(\mathrm{N}=11)$ went on to have an instrumental assessment in the form of Videofluoroscopy (VFS) or Fibreoptic Endoscopic Evaluation of Swallowing (FEES).

Mechanical oropharyngeal dysphagia, resulting in diet and/ or fluid modification, was identified in only 2 patients. Of those not formally assessed following case history, typical symptoms reported were in keeping with altered laryngeal sensitivity with no indications of aspiration.

At initial consultation, 8 patients were self-modifying their diet. Other relevant co-morbidities included reflux (71\%) and dysphonia (77\%).

Conclusions A high proportion of patients (52\%) with diagnosed ILO reported symptoms suggesting oropharyngeal 
dysphagia. Of these patients only $2.4 \%(n=2)$ were found to have oropharyngeal dysphagia on clinical assessment.

This preliminary study emphasises the importance of the SLT role within the Multi-Disciplinary Team; to identify whether these symptoms warrant further assessment. This study also highlights the benefits of access to instrumental assessment to prevent patient morbidity and inform diagnostic management.

Further prospective studies with larger patient cohorts may help to understand patient-reported symptoms of dysphagia further, and continue to inform clinical decision-making.

\section{Developing treatments for COVID-19}

\section{S48 LENZILUMAB EFFICACY AND SAFETY IN NEWLY HOSPITALIZED COVID-19 SUBJECTS: RESULTS FROM THE LIVE-AIR PHASE 3 RANDOMIZED DOUBLE-BLIND PLACEBO-CONTROLLED TRIAL}

\begin{abstract}
${ }^{1} \mathrm{Z}$ Temesgen, ${ }^{2} \mathrm{CD}$ Burger, ${ }^{3} \mathrm{~J}$ Baker, ${ }^{4} \mathrm{C}$ Polk, ${ }^{5} \mathrm{CR}$ Libertin, ${ }^{6} \mathrm{CF}$ Kelley, ${ }^{6} \mathrm{VC}$ Marconi, ${ }^{7} \mathrm{R}$ Orenstein, ${ }^{8} \mathrm{VM}$ Catterson, ${ }^{9} \mathrm{WS}$ Aronstein, ${ }^{10} \mathrm{C}$ Durrant, ${ }^{10} \mathrm{D}$ Chappell, ${ }^{10} \mathrm{O}$ Ahmed, ${ }^{10} \mathrm{G}$ Chappell, ${ }^{1} \mathrm{AD}$ Badley. ${ }^{1}$ Mayo Clinic, Division of Infectious Disease, Rochester, MN, USA; ${ }^{2}$ Mayo Clinic, Division of Pulmonary, Allergy and Sleep Medicine, Jacksonville, FL, USA; ${ }^{3}$ Hennepin Healthcare Research Institute, Minneapolis, MN, USA; ${ }^{4}$ Atrium Health, Charlotte, NC, USA; ${ }^{5}$ Mayo Clinic, Division of Infectious Diseases, Jacksonville, FL, USA; ${ }^{6}$ Division of Infectious Diseases, Emory University School of Medicine, Atlanta, GA, USA; ${ }^{7}$ Mayo Clinic Arizona, Division of Infectious Diseases, Phoenix, Arizona, USA; ${ }^{8}$ BioSymetrics, Inc., New York, NY, USA; ${ }^{9} C T$, Clinical Trial Services, Inc, Covington, KY, USA; ${ }^{10}$ Humanigen, Inc., Burlingame, CA, USA
\end{abstract}

\subsection{6/thorax-2021-BTSabstracts.54}

Background Severe COVID-19 pneumonia results from a hyperinflammatory immune response (cytokine storm, CS), characterized by GM-CSF mediated activation and trafficking of myeloid cells, leading to elevations of downstream inflammatory chemokines (MCP-1, IL-8, IP-10) and cytokines (IL-6, IL-1). CS leads to fever, hypotension, coagulopathy, respiratory failure, ARDS, and death. Lenzilumab is a novel Humaneered anti-human GM-CSF monoclonal antibody that binds GM-CSF and prevents signaling through its receptor. The LIVE-AIR Phase 3 randomized, double-blind, placebo-controlled trial investigated the efficacy and safety of lenzilumab to improve the likelihood of ventilator-free survival (referred to herein as survival without ventilation, SWOV), beyond standard supportive care, in hospitalized subjects with severe COVID-19.

Methods Subjects with COVID-19 $(n=520), \geq 18$ years, and $\leq 94 \%$ oxygen saturation on room air and/or requiring supplemental oxygen, but not invasive mechanical ventilation, were randomized to receive lenzilumab $(600 \mathrm{mg}, \mathrm{n}=261)$ or placebo $(n=259)$ via three intravenous infusions administered 8 hours apart. Subjects were followed through Day 28 following treatment.

Results Baseline demographics were comparable between the two treatment groups: male, 64.7\%; mean age, 60.5 years; mean BMI, $32.5 \mathrm{~kg} / \mathrm{m}^{2}$; median CRP, $79 \mathrm{mg} / \mathrm{L}$; CRP was $<150 \mathrm{mg} / \mathrm{L}$ in $78 \%$ of subjects. The most common comorbidities were hypertension (65.6\%), obesity (55.1\%), diabetes (53.4\%), chronic kidney disease (14.0\%), and coronary artery disease (13.6\%). Subjects received steroids (93.7\%), remdesivir $(72.4 \%)$, or both $(69.1 \%)$. Lenzilumab improved the likelihood of SWOV by $54 \%$ in the mITT population (HR: 1.54 ; 95\%CI: 1.02-2.32, $\mathrm{p}=0.0403)$. SWOV also relatively improved by $92 \%$ in the predefined subgroup of subjects who received both corticosteroids and remdesivir (1.92; 1.20-3.07; nominal $\mathrm{p}=0.0067)$. A key secondary endpoint of incidence of IMV, ECMO or death was also improved in patients receiving remdesivir $(p=0.020)$ or remdesivir and corticosteroids $(\mathrm{p}=0.0180)$. Serious adverse events were reported in $24.7 \%$ and $29.6 \%$ of the lenzilumab and placebo patients, respectively. Compared with placebo, lenzilumab produced no infusion-related reactions, and no attributable serious adverse events; including, hematologic or liver enzyme abnormalities, pulmonary alveolar proteinosis, or increased incidence of infection.

Conclusion Lenzilumab significantly improved SWOV in hypoxic COVID-19 patients upon hospitalization, with the greatest benefit observed in patients receiving treatment with remdesivir and corticosteroids. NCT04351152

\section{S49 C-REACTIVE PROTEIN AS A BIOMARKER FOR IMPROVED EFFICACY OF LENZILUMAB IN COVID-19 PATIENTS: RESULTS FROM THE LIVE-AIR TRIAL}

${ }^{1} \mathrm{Z}$ Temesgen, ${ }^{2} \mathrm{CD}$ Burger, ${ }^{3} \mathrm{~J}$ Baker, ${ }^{4} \mathrm{C}$ Polk, ${ }^{5} \mathrm{CR}$ Libertin, ${ }^{6} \mathrm{CF}$ Kelley, ${ }^{6} \mathrm{VC}$ Marconi,
${ }^{7} \mathrm{R}$ Orenstein, ${ }^{8} \mathrm{VM}$ Catterson, ${ }^{9} \mathrm{WS}$ Aronstein, ${ }^{10} \mathrm{C}$ Durrant, ${ }^{10} \mathrm{D}$ Chappell, ${ }^{10} \mathrm{O}$ Ahmed,
${ }^{10} \mathrm{G}$ Chappell, ${ }^{1} \mathrm{AD}$ Badley. ${ }^{1}$ Mayo Clinic, Division of Infectious Disease, Rochester, MN,
USA; ${ }^{2}$ Mayo Clinic, Division of Pulmonary, Allergy and Sleep Medicine, Jacksonville, FL,
USA; ${ }^{3}$ Hennepin Healthcare Research Institute Minneapolis, Minneapolis, MN, USA; ${ }^{4}$ Atrium
Health, Charlotte, NC, USA; ${ }^{5}$ Mayo Clinic, Division of Infectious Diseases, Jacksonville, FL,
USA; ${ }^{6}$ Division of Infectious Diseases, Emory University School of Medicine, Atlanta, GA,
USA; ${ }^{7}$ Mayo Clinic Arizona, Division of Infectious Diseases, Phoenix, Arizona, USA;
${ }^{8}$ BioSymetrics, Inc, New York, NY, USA; ${ }^{9} \mathrm{CTI}$, Clinical Trial Services, Inc, Covington, KY,
USA; ${ }^{10}$ Humanigen, Inc., Burlingame, CA, USA

10.1136/thorax-2021-BTSabstracts.55

Background The hyperinflammatory cytokine storm (CS) of COVID-19 is mediated by GM-CSF leading to release of downstream inflammatory chemokines, cytokines, and markers of systemic inflammation (C-reactive protein, CRP). The LIVE-AIR study demonstrated that lenzilumab, an anti-GMCSF monoclonal antibody in patients hospitalized with COVID-19, safely improved the likelihood of achieving the primary endpoint, survival without ventilation (SWOV) by 1.54-fold (HR: 1.54; 95\%CI: 1.02-2.32, $\mathrm{p}=0.0403$ ) compared with placebo. An exploratory analysis in patients with $\mathrm{CRP}<150 \mathrm{mg} / \mathrm{L}$ and age $<85$ years was conducted to determine lenzilumab efficacy when administered prior to advanced inflammation.

Methods LIVE-AIR was a phase 3 randomized, double-blind, placebo-controlled trial. Patients with COVID-19 $(n=520)$, $\geq 18$ years, and $\leq 94 \%$ oxygen saturation on room air and/or requiring supplemental oxygen, but not invasive mechanical ventilation (IMV), were randomized to receive lenzilumab (600 mg, $n=261)$ or placebo $(n=259)$ via three intravenous infusions administered 8 hours apart. Participants were followed through Day 28 following treatment.

Results Overall, baseline demographics were comparable between treatment groups: male, 64.7\%; mean age, 60.5 years; mean BMI, $32.5 \mathrm{~kg} / \mathrm{m}^{2}$; median CRP, $79 \mathrm{mg} / \mathrm{L}$; CRP was $<150 \mathrm{mg} / \mathrm{L}$ in $78 \%$ of participants. Participants received steroids (93.7\%), remdesivir (72.4\%), or both (69.1\%). Lenzilumab $(\mathrm{n}=159)$ improved the likelihood of SWOV by 3.04fold in participants with $\mathrm{CRP}<150 \mathrm{mg} / \mathrm{L}$ and age $<85$ years (3.04; 1.68-5.51, nominal $\mathrm{p}=0.0003)$ compared with placebo $(n=178)$. Response to lenzilumab was observed in the first through third quartiles of baseline CRP ( $<41 \mathrm{mg} / \mathrm{L}, \mathrm{HR}: 8.33$; 